EPJ Web of Conferences 73, 03011 (2014)

DOI: $10.1051 /$ epjconf/20147303011

(C) Owned by the authors, published by EDP Sciences, 2014

\title{
Higher bottomonia in the unquenched quark model
}

\author{
J. Ferretti ${ }^{1,2}$ and E. Santopinto ${ }^{2, a}$ \\ ${ }^{1}$ Instituto de Ciencias Nucleares, Universidad Nacional Autónoma de México, 04510 México DF, \\ México \\ ${ }^{2}$ INFN, Sezione di Genova, via Dodecaneso 33, 16146 Genova, Italy
}

\begin{abstract}
We show our results for the bottomonium spectrum with self energy corrections, due to the coupling to the meson-meson continuum. We also discuss our results for the open bottom strong decays of higher bottomonia in the ${ }^{3} P_{0}$ pair-creation model.
\end{abstract}

\section{Introduction}

Continuum coupling effects, due to the creation of $q \bar{q}$ pairs, determine a shift of the physical mass of a hadron with respect to its bare mass, as already shown by several authors in the baryon [1] and meson [2-6] sectors. For example, the resonance $\Lambda(1405)$ is strongly influenced by the nearby $\bar{K} N$ channel [7] and the $f_{0}(980)$ behaves remarkably as a $K \bar{K}$ molecule [8]. These continuum coupling (or pair-creation) effects, neglected in the naive quark model (QM) [9-14] (see also Refs. [15, 16]), are also important in the study of other observables, such as the importance of the orbital angular momentum in the spin of the proton [17], the flavor asymmetry of the proton [18] and the strange content of the electromagnetic form factors of the nucleon [19]. Important informations on mesons are also provided by their strong, electromagnetic and weak decay modes. In particular, the open bottom strong decays are transitions to open bottom final states, where the initial $b \bar{b}$ meson decays by $q \bar{q}$ pair-production $(q=u, d$ or $s)$ into a pair of $b \bar{q}$ and $q \bar{b}$ mesons. Since the QCD mechanism behind the OZI-allowed strong decays is still not clear, several phenomenological models have been developed to carry on this type of studies: they include pair-creation models [20], elementary meson emission models and effective Lagrangian approaches. Attempts at modeling strong decays within the quark model (QM) formalism date from Micu's suggestion [20], that hadron decays proceed through $q \bar{q}$ pair production with vacuum quantum numbers, i.e. $J^{P C}=0^{++}$. Since the $q \bar{q}$ pair corresponds to a ${ }^{3} P_{0}$ quark-antiquark state, this is now generally known as the ${ }^{3} P_{0}$ pair-creation model [20].

In this contribution, we show an application of the unquenched quark model (UQM) [4-6, 17-19] to the calculation of the bottomonium spectrum with self energy corrections [6]. We also discuss our results for the open bottom strong decay widths of higher bottomonia [6], computed within a modified version of the ${ }^{3} P_{0}$ pair-creation model [4-6].

\footnotetext{
ae-mail: santopinto@ge.infn.it
}

This is an Open Access article distributed under the terms of the Creative Commons Attribution License 4.0, which permits unrestricted use, distribution, and reproduction in any medium, provided the original work is properly cited. 
Table 1. Self $\left[\Sigma\left(E_{a}\right)\right]$ and bare $\left(E_{a}\right)$ energies of the $\eta_{b}$ state (in $\mathrm{MeV}$ ), whose sum gives the physical mass $M_{a}$ of the meson [6]. This value is compared to the corresponding experimental data [21].

\begin{tabular}{cccccccccccc}
\hline State & $B \bar{B}^{*}$ & $B^{*} \bar{B}^{*}$ & $B_{s} \bar{B}_{s}^{*}$ & $B_{s}^{*} \bar{B}_{s}^{*}$ & $B_{c} \bar{B}_{c}^{*}$ & $B_{c}^{*} \bar{B}_{c}^{*}$ & $\Upsilon \Upsilon$ & $\Sigma\left(E_{a}\right)$ & $E_{a}$ & $M_{a}$ & $M_{\text {exp. }}$ \\
& $\bar{B} B^{*}$ & & $\bar{B}_{s} B_{s}^{*}$ & & $\bar{B}_{c} B_{c}^{*}$ & & & & & & \\
\hline$\eta_{b}\left(1^{3} S_{1}\right)$ & -26 & -26 & -5 & -5 & -1 & -1 & 0 & -64 & 9455 & 9391 & 9391 \\
\hline
\end{tabular}

\section{Self energies in the UQM}

The Hamiltonian of the UQM [4-6, 17-19] is

$$
H=H_{0}+V
$$

where the first term, $H_{0}$, acts only in the bare meson space and the second term, $V$, couples a meson state $|A\rangle$ to the meson-meson continuum $|B C\rangle$. In the UQM approach [4-6, 17-19], which is a generalization of the unitarized quark model by Törnqvist and Zenczykowski [1], the effects of $q \bar{q}$ pairs are introduced explicitly into the $\mathrm{QM}$ through a ${ }^{3} P_{0}$ pair-creation mechanism.

The dispersive equation for the self energy $\Sigma\left(E_{a}\right)$ in the UQM is given by

$$
\begin{aligned}
\Sigma\left(E_{a}\right) & =\sum_{B C} \int_{0}^{\infty} q^{2} d q \frac{\left|V_{a, b c}(q)\right|^{2}}{E_{a}-E_{b c}} \\
& =\sum_{B C, \ell, J} \int_{0}^{\infty} q^{2} d q \frac{\left|\left\langle B C \vec{q} \ell J\left|T^{\dagger}\right| A\right\rangle\right|^{2}}{E_{a}-E_{b c}}
\end{aligned}
$$

and the bare energy $E_{a}$, computed within the relativized QM for mesons [11], satisfies:

$$
M_{a}=E_{a}+\Sigma\left(E_{a}\right)
$$

Here, $M_{a}$ is the physical mass of the meson $A, B$ and $C$ are the intermediate state mesons, with energies $E_{b}=\sqrt{M_{b}^{2}+q^{2}}$ and $E_{c}=\sqrt{M_{c}^{2}+q^{2}}, \vec{q}$ and $\ell$ the relative radial momentum and orbital angular momentum between $B$ and $C$, and $\vec{J}=\vec{J}_{b}+\vec{J}_{c}+\vec{\ell}$ is the total angular momentum. The wave functions of the mesons $A, B$ and $C$ are written as harmonic oscillator wave functions, depending on a single oscillator parameter $\alpha=0.5 \mathrm{GeV}$ [4-6, 17-19]. The self energies $\Sigma\left(E_{a}\right)$ 's of Eq. (2) are computed summing over a complete set of accessible $\mathrm{SU}_{\mathrm{f}}(4) \otimes \mathrm{SU}_{\text {spin }}(2) 1 S$ intermediate states. If the bare energy of the initial meson $A, E_{a}$, is above the threshold $B C$, the self energy correction due to the channel $B C$ is given by

$$
\begin{aligned}
\Sigma\left(E_{a}\right)(B C) & =\mathcal{P} \int_{M_{b}+M_{c}}^{\infty} \frac{d E_{b c}}{E_{a}-E_{b c}} \frac{q E_{b} E_{c}}{E_{b c}}\left|\left\langle B C \vec{q} \ell J\left|T^{\dagger}\right| A\right\rangle\right|^{2} \\
& +2 \pi i\left\{\frac{q E_{b} E_{c}}{E_{a}}\left|\left\langle B C \vec{q} \ell J\left|T^{\dagger}\right| A\right\rangle\right|^{2}\right\}_{E_{b c}=E_{a}},
\end{aligned}
$$

where the symbol $\mathcal{P}$ stands for a principal part integral and $2 \pi i\left\{\frac{q E_{b} E_{c}}{E_{a}}\left|\left\langle B C \vec{q} \ell J\left|T^{\dagger}\right| A\right\rangle\right|^{2}\right\}_{E_{b c}=E_{a}}$ is the imaginary part of the self energy.

Finally, in Table 1, we show our UQM result for the mass of the $\eta_{b}(1 S)$ resonance with self energy corrections [6]. It is interesting to observe that the contributions to the self energy due to unphysical heavy $Q \bar{Q}$ pair-creation $(Q=c, b)$ are strongly suppressed, by substituting the pair-creation strength of the ${ }^{3} P_{0}$ model, $\gamma_{0}$, with an effective one, $\gamma_{0}^{\text {eff }}[3-6]$. 
Table 2. Our results of Ref. [6] for $\Upsilon(4 S), \Upsilon(10860)$ and $\Upsilon(11020)$ are compared to the experimental data [21, 23]. The results are expressed in $\mathrm{MeV}$.

\begin{tabular}{cccc}
\hline State & $\Gamma\left({ }^{3} P_{0}\right)[6]$ & $\Gamma_{\exp }(\mathrm{PDG})[21]$ & $\Gamma_{\exp }(\mathrm{BABAR})[23]$ \\
\hline$\Upsilon(4 S)$ & 21 & $21 \pm 3$ & - \\
$\Upsilon(10860)$ & 71 & $43 \pm 22$ & $58 \pm 3$ \\
$\Upsilon(11020)$ & 36 & $79 \pm 16$ & $37 \pm 3$ \\
\hline
\end{tabular}

\section{Open bottom strong decays in the ${ }^{3} P_{0}$ pair-creation model}

In this section, we discuss our results for the open bottom strong decay widths of higher bottomonia $[6,22]$. The decay widths are calculated as $[4-6,20]$

$$
\Gamma_{A \rightarrow B C}=\Phi_{A \rightarrow B C}\left(q_{0}\right) \sum_{\ell, J}\left|\left\langle B C \vec{q}_{0} \ell J\left|T^{\dagger}\right| A\right\rangle\right|^{2},
$$

where $\Phi_{A \rightarrow B C}\left(q_{0}\right)$ stands for the relativistic phase space factor [4-6, 20],

$$
\Phi_{A \rightarrow B C}=2 \pi q_{0} \frac{E_{b}\left(q_{0}\right) E_{c}\left(q_{0}\right)}{M_{a}},
$$

that depends on the relative momentum $q_{0}$ between $B$ and $C$ and on the energies of the two intermediate state mesons, $E_{b}=\sqrt{M_{b}^{2}+q_{0}^{2}}$ and $E_{c}=\sqrt{M_{c}^{2}+q_{0}^{2}}$.

Finally, in Table 2 we compare some of our results of Ref. [6] to the experimental data [21,23].

\section{Continuum components and decay widths in the UQM}

The ${ }^{3} P_{0}$ model coupling $T^{\dagger}$ of Eqs. (2) and (4) gives rise to a continuum component in an initial valence state $|A\rangle$. Its norm, providing the probability that the physical energy eigenstate $\left|\Psi_{A}\right\rangle$ is in the mesonmeson continuum, can be written as [4]:

$$
P_{a}^{s e a}=\sum_{B C \ell J} \int_{0}^{\infty} q^{2} d q \frac{\left|\left\langle B C \vec{q} \ell J\left|T^{\dagger}\right| A\right\rangle\right|^{2}}{\left(E_{a}-E_{b}-E_{c}\right)^{2}},
$$

where one has to sum over virtual channels $B C$. The probability to find $\left|\Psi_{A}\right\rangle$ in its valence component $|A\rangle$ is then $P_{b \bar{b}}=1-P_{a}^{\text {sea }}$. One can also relate the imaginary part of the self energy of Eq. (4),

$$
\operatorname{IM}\left[\Sigma\left(E_{a} ; B C\right)\right]=2 \pi i\left\{\frac{q E_{b} E_{c}}{E_{a}}\left|\left\langle B C \vec{q} \ell J\left|T^{\dagger}\right| A\right\rangle\right|^{2}\right\}_{E_{b c}=E_{a}},
$$

to the decay width by [4]

$$
\Gamma_{A \rightarrow B C}=\operatorname{Im}\left[\Sigma\left(E_{a}\right)(B C)\right] .
$$

In a subsequent paper, we intend to calculate the continuum components of higher bottomonium states through Eq. (7) and compare our ${ }^{3} P_{0}$ model results [6] for the open bottom strong decays to those obtained via Eq. (9). This could be particularly interesting in the case of $\chi_{b}(3 P)$ mesons, that may contain important continuum components $[6,22]$.

\section{Conclusions}

We discussed the results of an UQM [4-6, 17-19] calculation of the spectrum of $b \bar{b}$ mesons with self energy corrections [6], due to the coupling to the meson-meson continuum. Even if the self energy corrections to the spectrum of bottomonia [6] are relatively small (1-2\% of the corresponding meson mass), these effects can become qualitatively important in the case of suspected non $b \bar{b}$ states, such as 
$\chi_{b}(3 P)$ mesons $[24,25]$, that are states close to the first open bottom decay thresholds. In Ref. [6], we have shown that in the case of $\chi_{b}(3 P)$ mesons the self energy corrections give rise to $B \bar{B}, B \bar{B}^{*}$ and $B^{*} \bar{B}^{*}$ components in addition to the $b \bar{b}$ core [6]. Then, we discussed our results for the open bottom strong decay widths of $b \bar{b}$ states [6], computed within a modified version of the ${ }^{3} P_{0}$ pair-creation model [4-6]. Finally, we analysed a possible application of the UQM formalism to the calculation of the continuum components and the open bottom strong decays of higher bottomonia, that could be especially interesting in the case of $\chi_{b}(3 P)$ mesons.

\section{References}

[1] N.A. Törnqvist and P. Zenczykowski, Phys. Rev. D 29, 2139 (1984); Z. Phys. C 30, 83 (1986)

[2] S. Ono and N.A. Törnqvist, Z. Phys. C 23, 59 (1984); K. Heikkila et al., Phys. Rev. D 29, 110 (1984); S. Ono et al., Phys. Rev. D 34, 186 (1986)

[3] Y.S. Kalashnikova, Phys. Rev. D 72, 034010 (2005)

[4] J. Ferretti, G. Galatá, E. Santopinto and A. Vassallo, Phys. Rev. C 86, 015204 (2012)

[5] J. Ferretti, G. Galatá and E. Santopinto, Phys. Rev. C 88, 015207 (2013)

[6] J. Ferretti and E. Santopinto, arXiv:1306.2874 [hep-ph]

[7] R.H. Dalitz and S.F. Tuan, Phys. Rev. Lett. 2, 425 (1959)

[8] S.M. Flatte et al., Phys. Lett. B 38, 232 (1972)

[9] E. Eichten et al., Phys. Rev. Lett. 34, 369 (1975); E. Eichten et al., Phys. Rev. D 17, 3090 (1978); 21, 203 (1980)

[10] N. Isgur and G. Karl, Phys. Rev. D 18, 4187 (1978)

[11] S. Godfrey and N. Isgur, Phys. Rev. D 32, 189 (1985); S. Capstick and N. Isgur, Phys. Rev. D 34, 2809 (1986)

[12] M.M. Giannini and E. Santopinto, Phys. Rev. C 49, 1258 (1994); M. Ferraris et al., Phys. Lett. B 364, 231 (1995); E. Santopinto, F. Iachello and M.M. Giannini, Nucl. Phys. A 623, 100C (1997); Eur. Phys. J. A 1, 307 (1998); M. Aiello, M.M. Giannini and E. Santopinto, J. Phys. G 24, 753 (1998); R. Bijker, F. Iachello and E. Santopinto, J. Phys. A 31, 9041 (1998); M.D. Sanctis et al., Phys. Rev. C 62, 025208 (2000); M.M. Giannini, E. Santopinto and A. Vassallo, Eur. Phys. J. A 12, 447 (2001); Nucl. Phys. A 699, 308 (2002); L. Tiator et al., Eur. Phys. J. A 19, 55 (2004); M. Gorchtein et al., Phys. Rev. C 70, 055202 (2004); M. De Sanctis et al., Eur. Phys. J. A 19, 81 (2004); Nucl. Phys. A 755, 294 (2005); Phys. Rev. C 76, 062201 (2007); E. Santopinto and M.M. Giannini, Phys. Rev. C 86, 065202 (2012)

[13] E. Santopinto, Phys. Rev. C 72, 022201 (2005); J. Ferretti, A. Vassallo and E. Santopinto, Phys. Rev. C 83, 065204 (2011); M. De Sanctis et al., Phys. Rev. C 84, 055201 (2011)

[14] E. Santopinto and G. Galatá, Phys. Rev. C 75, 045206 (2007)

[15] P. Guo et al., Phys. Rev. D 77, 056005 (2008); 78, 056003 (2008)

[16] S. Capstick et al., Eur. Phys. J. A 35, 253 (2008)

[17] R. Bijker and E. Santopinto, Phys. Rev. C 80, 065210 (2009); E. Santopinto and R. Bijker, Few Body Syst. 44, 95 (2008)

[18] E. Santopinto and R. Bijker, Phys. Rev. C 82, 062202 (2010)

[19] R. Bijker, J. Ferretti and E. Santopinto, Phys. Rev. C 85, 035204 (2012)

[20] L. Micu, Nucl. Phys. B 10, 521 (1969); A. Le Yaouanc et al., Phys. Rev. D 8, 2223 (1973)

[21] J. Beringer et al. [Particle Data Group Collaboration], Phys. Rev. D 86, 010001 (2012)

[22] J. Ferretti, G. Galatà and E. Santopinto, arXiv:1401.4431 [nucl-th]

[23] B. Aubert et al. [BaBar Collaboration], Phys. Rev. Lett. 102, 012001 (2009)

[24] G. Aad et al. [ATLAS Collaboration], Phys. Rev. Lett. 108, 152001 (2012)

[25] V.M. Abazov et al. [D0 Collaboration], Phys. Rev. D 86, 031103 (2012) 